\title{
On the prolate spheroidal wave functions and Hardy's uncertainty principle
}

\author{
Elmar Pauwels * Maurice de Gosson ${ }^{\dagger}$
}

\begin{abstract}
We prove a weak version of Hardy's uncertainty principle using properties of the prolate spheroidal wave functions (PSWFs). We describe the eigenvalues of the sum of a time limiting operator and a band limiting operator acting on $L^{2}(\mathbb{R})$. A weak version of Hardy's uncertainty principle follows from the asymptotic behavior of the largest eigenvalue as the time limit and the band limit approach infinity. An asymptotic formula for this eigenvalue is obtained from its well-known counterpart for the prolate integral operator.
\end{abstract}

\section{Introduction}

Classical Hardy's uncertainty principle is formulated as follows.

Theorem 1.1. Let $a, b, M>0$, and let $f$ be a measureable function on $\mathbb{R}$ such that

$$
|f(x)| \leqslant M e^{-a x^{2} / 2},
$$

and

$$
|\hat{f}(\xi)| \leqslant M e^{-b \xi^{2} / 2},
$$

for all $x, \xi \in \mathbb{R}$. If $a b>1$, then $f=0$.

*NuHAG, Faculty of Mathematics, University of Vienna, elmar.pauwels@univie.ac.at

${ }^{\dagger}$ Corresponding author, NuHAG, Faculty of Mathematics, University of Vienna, maurice.de.gosson@univie.ac.at 
Several proofs of this classical theorem are known e.g. [2, 3, 4, 9]. Typically, they use methods of complex analysis, and rely on somewhat indirect arguments. Our objective is to give a new and direct proof with methods of real analysis, however only of the following weaker result.

Theorem 1.2. Let $a, b, M>0$, and let $f$ be a measureable function on $\mathbb{R}$ such that

$$
|f(x)| \leqslant M e^{-a x^{2} / 2}
$$

and

$$
|\hat{f}(\xi)| \leqslant M e^{-b \xi^{2} / 2}
$$

for all $x, \xi \in \mathbb{R}$. If $a b \geqslant 4$, then $f=0$.

We prove this weak version of Hardy's uncertainty principle using properties of the prolate spheroidal wave functions (PSWFs), which appear e.g. in a solution of the concentration problem for bandlimited functions [7]. First, we describe the spectrum of the sum of a time limiting operator and a band limiting operator acting on $L^{2}(\mathbb{R})$. Specifically, we express the spectrum in terms of the eigenvalues of the prolate integral operator.

Then we derive the weak version of Hardy's uncertainty principle from the asymptotic behavior of the largest eigenvalue of the sum of the time and band limiting operators as the time limit and the band limit approach infinity. An asymptotic formula for this eigenvalue is obtained from its well-known counterpart for the prolate integral operator.

Our approach reveals a relationship between Hardy's uncertainty principle and the theory of bandlimited functions.

\section{Mathematical preliminaries}

The Fourier transform of a function $f \in \boldsymbol{L}^{2}(\mathbb{R})$ is a bounded operator on $\boldsymbol{L}^{2}(\mathbb{R})$ defined as follows:

$$
\mathcal{F} f(\xi)=\frac{1}{\sqrt{2 \pi}} \lim _{N \rightarrow \infty} \int_{-N}^{N} e^{-i x \xi} f(x) d x,
$$

where the limit is taken in $\boldsymbol{L}^{2}(\mathbb{R})$. We also use the notation $\hat{f}$ for $\mathcal{F} f$.

$\mathcal{F}$ is invertible on $\boldsymbol{L}^{2}(\mathbb{R})$, and its bounded inverse, the inverse Fourier transform, is defined as follows: 


$$
\mathcal{F}^{-1} g(x)=\frac{1}{\sqrt{2 \pi}} \lim _{N \rightarrow \infty} \int_{-N}^{N} e^{i \xi x} g(\xi) d \xi
$$

Consequently,

$$
\mathcal{F}^{-1}=\mathcal{F}^{*},
$$

i.e. the Fourier transform is a unitary operator on $\boldsymbol{L}^{2}(\mathbb{R})$.

A bounded operator $P$ on a Hilbert space is called idempotent, if

$$
P^{2}=P
$$

A bounded operator $P$ on a Hilbert space is called an orthogonal projection, if it is idempotent and Hermitian, i.e.

$$
P^{2}=P \quad \text { and } \quad P^{*}=P .
$$

We denote the characteristic function of a set $E \subset \mathbb{R}$ by $\chi_{E}$

$$
\chi_{E}(x)= \begin{cases}1, & \text { if } x \in E \\ 0, & \text { otherwise }\end{cases}
$$

For a fixed set $E \subset \mathbb{R}$, the mapping $f \mapsto \chi_{E} f$ is an orthogonal projection on $\boldsymbol{L}^{2}(\mathbb{R})$. We denote this projection also by $\chi_{E}$. In particular, we use the notation $\chi_{(-\tau, \tau)}$, when $E=(-\tau, \tau), \tau>0$.

For a fixed $\omega>0$, we define the operator $S_{\omega}$ as follows

$$
S_{\omega}:=\mathcal{F} \chi_{(-\omega, \omega)} \mathcal{F}^{*}
$$

The operator $S_{\omega}$ is also an orthogonal projection on $\boldsymbol{L}^{2}(\mathbb{R})$.

The integral kernel of $S_{\omega}$ is well known, namely

$$
S_{\omega} f(x)=\int_{\mathbb{R}} \frac{\sin \omega(x-y)}{\pi(x-y)} f(y) d y .
$$

This kernel is computed as follows:

$$
k(x, y)=\frac{1}{2 \pi} \int_{-\omega}^{\omega} e^{-i x t} e^{i t y} d t=\frac{1}{\pi} \frac{\sin \omega(x-y)}{x-y} .
$$

Due to (11), $S_{\omega}$ is also an orthogonal projection on $\boldsymbol{L}^{2}(\mathbb{R})$. 
If $P$ is an orthogonal projection, then

$$
\begin{aligned}
\left(I-P^{*}\right)(I-P) & =(I-P)^{2} \\
& =I-2 P+P^{2} \\
& =I-P .
\end{aligned}
$$

Thus for every vector $f$,

$$
\begin{aligned}
\|(I-P) f\|^{2} & =\left\langle\left(I-P^{*}\right)(I-P) f, f\right\rangle \\
& =\langle(I-P) f, f\rangle .
\end{aligned}
$$

For a bounded operator $T, \sigma(T)$ denotes the spectrum of $T$. We need the following well-known lemma, see [1, Prop. 6, p. 16].

Lemma 2.1. Let $A$ and $B$ be bounded operators on a Hilbert space. For $\lambda \neq 0, \lambda \in \sigma(A B)$ if and only if $\lambda \in \sigma(B A)$.

The following lemma has a straightforward proof, which is omitted.

Lemma 2.2. If $P$ is an idempotent bounded operator on a Hilbert space and $\lambda \neq 0,1$, then

$$
(\lambda I-P)^{-1}=\frac{1}{\lambda} I+\frac{1}{\lambda(\lambda-1)} P .
$$

\section{Spectrum of $\chi_{(-\tau, \tau)}+S_{\omega}$}

In this section, we describe the spectrum of the operator $\chi_{(-\tau, \tau)}+S_{\omega}$ on $\boldsymbol{L}^{2}(\mathbb{R})$, where $\tau, \omega>0$.

For a fixed $c>0$, the integral operator on $\boldsymbol{L}^{2}(-1,1)$ with the kernel

$$
\frac{\sin c(x-y)}{\pi(x-y)}
$$

has eigenvalues $\lambda_{0}>\lambda_{1}>\cdots>0$ [7, 8]. The eigenfunctions are the PSWFs, and the eigenvalues obey certain asymptotic formulas. We are only interested in the largest eigenvalue $\lambda_{0}$ which has the following asymptotics [8]

$$
\lambda_{0}=1-4 \sqrt{\pi} \sqrt{c} e^{-2 c}\left(1+\mathcal{O}\left(\frac{1}{c}\right)\right),
$$

when $c \rightarrow \infty$. 
We show that the eigenvalues of $T=\chi_{(-\tau, \tau)}+S_{\omega}$, acting on $\boldsymbol{L}^{2}(\mathbb{R})$, can be expressed in terms of those of the operator with kernel (13), acting not on $\boldsymbol{L}^{2}(\mathbb{R})$, but rather on $\boldsymbol{L}^{2}(-\tau, \tau)$.

To indicate the dependence on $c$, we write $\lambda_{n}(c)$.

Theorem 3.1. Let $\tau, \omega>0$ and let $T=\chi_{(-\tau, \tau)}+S_{\omega}$. If $\lambda \in \sigma(T)$ and $\lambda \neq 0,1$, then

$$
(\lambda-1)^{2}=\lambda_{n}(\omega \tau)
$$

for some $n$.

Proof. In this proof we can assume that $\tau=1$. The general case follows by a linear change of variables.

It follows from the assumptions that $\lambda I-\chi_{(-1,1)}-S_{\omega}$ is singular, and so is the operator

$$
\begin{aligned}
\left(\lambda I-S_{\omega}\right)^{-1}\left(\lambda I-\chi_{(-1,1)}-S_{\omega}\right) & =I-\left(\lambda I-S_{\omega}\right)^{-1} \chi_{(-1,1)} \\
& =I-\left(\frac{1}{\lambda} I+\frac{1}{\lambda(1-\lambda)} S_{\omega}\right) \chi_{(-1,1)},
\end{aligned}
$$

where we used Lemma 2.2 for $\left(\lambda I-S_{\omega}\right)^{-1}$. Thus

$$
\begin{aligned}
\lambda & \in \sigma\left(\chi_{(-1,1)}+\frac{1}{\lambda-1} S_{\omega} \chi_{(-1,1)}\right) \\
& =\sigma\left(\chi_{(-1,1)}+\frac{1}{\lambda-1} S_{\omega} \chi_{(-1,1)}^{2}\right) .
\end{aligned}
$$

We use Lemma 2.1 for the operators $\chi_{(-1,1)}$ and $I+\frac{1}{\lambda-1} S_{\omega} \chi_{(-1,1)}$ and the assumption that $\lambda \neq 0$, to conclude that

$$
\lambda \in \sigma\left(\chi_{(-1,1)}+\frac{1}{\lambda-1} \chi_{(-1,1)} S_{\omega} \chi_{(-1,1)}\right) .
$$

It follows that the operator

$$
\lambda I-\chi_{(-1,1)}-\frac{1}{\lambda-1} \chi_{(-1,1)} S_{\omega} \chi_{(-1,1)}
$$

is singular, and so is

$$
I-\left(\lambda I-\chi_{(-1,1)}\right)^{-1} \frac{1}{\lambda-1} \chi_{(-1,1)} S_{\omega} \chi_{(-1,1)} .
$$


The operator $\chi_{(-1,1)} S_{\omega} \chi_{(-1,1)}$ is Hilbert-Schmidt, and therefore compact, and so is $\left(\lambda I-\chi_{(-1,1)}\right)^{-1} \chi_{(-1,1)} S_{\omega} \chi_{(-1,1)}$.

From the spectral theory of compact operators, it follows that 1 is an eigenvalue of

$$
\left(\lambda I-\chi_{(-1,1)}\right)^{-1} \frac{1}{\lambda-1} \chi_{(-1,1)} S_{\omega} \chi_{(-1,1)},
$$

i.e. there is an $f \in \boldsymbol{L}^{2}(\mathbb{R}), f \neq 0$ such that

$$
f-\left(\lambda I-\chi_{(-1,1)}\right)^{-1} \frac{1}{\lambda-1} \chi_{(-1,1)} S_{\omega} \chi_{(-1,1)} f=0
$$

or

$$
\lambda f-\chi_{(-1,1)} f-\frac{1}{\lambda-1} \chi_{(-1,1)} S_{\omega} \chi_{(-1,1)} f=0 .
$$

Using (32), we note that $\chi_{(-1,1)} f \neq 0$ in $\boldsymbol{L}^{2}(\mathbb{R})$, because $\lambda f \neq 0$ in $\boldsymbol{L}^{2}(\mathbb{R})$. We now multiply (32) by $\chi_{(-1,1)}$ on the left to get

$$
\lambda \chi_{(-1,1)} f-\chi_{(-1,1)} f-\frac{1}{\lambda-1} \chi_{(-1,1)} S_{\omega} \chi_{(-1,1)} f=0
$$

and, consequently

$$
(\lambda-1)^{2} \chi_{(-1,1)} f-\chi_{(-1,1)} S_{\omega} \chi_{(-1,1)} f=0 .
$$

Thus $\chi_{(-1,1)} f$ is an eigenfunction of the operator with kernel $\frac{\sin \omega(x-y)}{\pi(x-y)}$ on $\boldsymbol{L}^{2}(-1,1)$ with eigenvalue $(\lambda-1)^{2}$.

Thus

$$
(\lambda-1)^{2}=\lambda_{n}(\omega)
$$

for some $n$.

Remark 3.2. Only Theorem 3.1 is used in the proof of Theorem 1.2. However, we devote the rest of this section to a complete description of the spectrum of the operator $T=\chi_{(-\tau, \tau)}+S_{\omega}$.

In this proof we can again assume that $\tau=1$. The general case follows by a linear change of variables.

We can show that if $(\lambda-1)^{2}=\lambda_{n}(\omega)$, then there exists an eigenfunction for $T$ with eigenvalue $\lambda$.

It follows from Slepian's theory, that $0<\lambda_{n}(\omega)<1$. We write

$$
f=\psi_{n}+(\lambda-1) \widetilde{\psi}_{n}
$$


where $\psi_{n}$ is the $n$th PSWF and $\widetilde{\psi}_{n}$ is the extension of $\psi_{n}$ to $\mathbb{R}$, i.e.

$$
S_{\omega} \psi_{n}=\lambda_{n}(\omega) \widetilde{\psi}_{n}
$$

and

$$
\chi_{(-1,1)} \widetilde{\psi}_{n}=\psi_{n}
$$

We multiply (36) by $\chi_{(-1,1)}$ to get

$$
\chi_{(-1,1)} f=\psi_{n}+(\lambda-1) \psi_{n}=\lambda \psi_{n} .
$$

Moreover,

$$
\begin{aligned}
\left(\lambda I-S_{\omega}\right)^{-1} \chi_{(-1,1)} f & =\left(\frac{1}{\lambda} I+\frac{1}{\lambda(\lambda-1)} S_{\omega}\right) \lambda \psi_{n} \\
& =\psi_{n}+\frac{1}{\lambda-1} \lambda_{n} \widetilde{\psi}_{n} \\
& =\psi_{n}+(\lambda-1) \widetilde{\psi}_{n} \\
& =f
\end{aligned}
$$

where we used the assumption that $\lambda_{n}=(\lambda-1)^{2}$.

Finally,

$$
\chi_{(-1,1)} f=\left(\lambda I-S_{\omega}\right) f
$$

or

$$
\chi_{(-1,1)} f+S_{\omega} f=\lambda f .
$$

Thus all numbers of the form $1 \pm \sqrt{\lambda_{n}(\omega)}, n=0,1, \ldots$ are eigenvalues of $T$.

The point $\lambda=1$ is also in the spectrum $\sigma(T)$ as an accumulation point of the eigenvalues.

It remains to consider $\lambda=0$. To observe that $T$ is singular, we consider the sequence of functions

$$
f_{n}(x)=e^{i n x} e^{-(x-n)^{2}}
$$

where $x \in \mathbb{R}$. It is clear that

$$
\left\|f_{n}\right\|=\left\|f_{0}\right\|>0
$$

for $n=0,1, \ldots$, but

$$
\left|T f_{n}\right| \rightarrow 0
$$


in $\boldsymbol{L}^{2}(\mathbb{R})$.

Thus we have shown that $\sigma(T)$ consists of the eigenvalues of $T$ of the form $1 \pm \sqrt{\lambda_{n}(\omega)}, n=0,1, \ldots$, and two additional points $\lambda=0$ and $\lambda=1$.

The spectrum of the sum of two orthogonal projections was described in [6] in a somewhat different setting.

\section{Proof of the Theorem 1.2}

In this section, we present the proof of Theorem 1.2.

Proof of Theorem 1.2. In this proof, we assume that

$$
a=b=2 .
$$

The general case follows by a linear change of variables.

For a fixed $\tau>0$, we consider the restriction $\chi_{(-\tau, \tau)} f$ of $f$ to the interval $(-\tau, \tau)$. The decay of $f$ at infinity in (3) gives an estimate on $f-\chi_{(-\tau, \tau)} f$ in the $\boldsymbol{L}^{2}$-norm. Specifically,

$$
\begin{aligned}
\left\|f-\chi_{(-\tau, \tau)} f\right\|^{2} & =\int_{|x|>\tau}|f(x)|^{2} d x \\
& \leqslant M^{2} \int_{|x|>\tau}\left(e^{-a x^{2} / 2}\right)^{2} d x \\
& =2 M^{2} \int_{\tau}^{\infty} e^{-a x^{2}} d x \\
& \leqslant 2 M^{2} \int_{\tau}^{\infty} \frac{x}{\tau} e^{-a x^{2}} d x \\
& =\frac{M^{2}}{a \tau} e^{-a \tau^{2}} .
\end{aligned}
$$

Similarly, (44) implies that for a fixed $\omega>0$,

$$
\left\|f-S_{\omega} f\right\|^{2} \leqslant \frac{M^{2}}{b \omega} e^{-b \omega^{2}} .
$$


Setting $\tau=\omega$, using (18) and combining (49), (54) and (55) gives

$$
\begin{aligned}
\| f & -\chi_{(-\tau, \tau)} f\left\|^{2}+\right\| f-S_{\omega} f \|^{2}= \\
& =\left\langle\left(I-\chi_{(-\tau, \tau)}\right) f, f\right\rangle+\left\langle\left(I-S_{\omega}\right) f, f\right\rangle \\
& =\left\langle\left(2 I-\chi_{(-\tau, \tau)}-S_{\omega}\right) f, f\right\rangle \\
& \leqslant \frac{M^{2}}{\omega} e^{-2 \omega^{2}} .
\end{aligned}
$$

The operator $T^{\prime}=2 I-\chi_{(-\tau, \tau)}-S_{\omega}$ is Hermitian. According to Theorem 3.1, its smallest eigenvalue $\lambda_{\min }$ satisfies

$$
\lambda_{\text {min }} \geqslant 2-\left(1+\sqrt{\lambda_{0}}\right)=1-\sqrt{\lambda_{0}} .
$$

Consequently,

$$
\begin{aligned}
\left(1-\sqrt{\lambda_{0}}\right)\|f\|^{2} & \leqslant \lambda_{\min }\|f\|^{2} \\
& \leqslant\left\langle\left(2 I-\chi_{(-\tau, \tau)}-S_{\omega}\right) f, f\right\rangle \\
& \leqslant \frac{M^{2}}{\omega} e^{-2 \omega^{2}} .
\end{aligned}
$$

The eigenvalue $\lambda_{0}$ satisfies (21). Thus since $c=\omega \tau=\omega^{2}$, we get

$$
\lambda_{0}=1-4 \sqrt{\pi} \omega e^{-2 \omega^{2}}\left(1+\mathcal{O}\left(\frac{1}{\omega^{2}}\right)\right) .
$$

We recall the elementary formula

$$
\sqrt{1-x}=1-x / 2+\mathcal{O}\left(x^{2}\right)
$$

as $x \rightarrow 0$. Substituting (64) and (65) into (63) we obtain

$$
2 \sqrt{\pi} \omega e^{-2 \omega^{2}}\|f\|^{2}\left(1+\mathcal{O}\left(\frac{1}{\omega^{2}}\right)\right) \leqslant \frac{M^{2}}{\omega} e^{-2 \omega^{2}} .
$$

Letting $\omega \rightarrow \infty$, we deduce that $\|f\|=0$.

\subsection{Alternative proof}

A reviewer of this paper has remarked that an alternative proof is possible based on the following result proved in [5, p. 68]. 
Theorem 4.1. If $\|f\|=1$,

$$
\begin{aligned}
& \alpha=\left(\int_{-\frac{T}{2}}^{\frac{T}{2}}|f(t)|^{2} d t\right)^{\frac{1}{2}}, \\
& \beta=\left(\int_{-\Omega}^{\Omega}|\hat{f}(\xi)|^{2} d \xi\right)^{\frac{1}{2}},
\end{aligned}
$$

then

$$
\arccos \alpha+\arccos \beta \geqslant \arccos \sqrt{\lambda_{0}\left(\frac{1}{2} \Omega T\right)} .
$$

We present an outline of an alternative proof. Let us assume that $\|f\|=1$. It follows from (49) and (54) that

$$
\left\|\chi_{(-\tau, \tau)} f\right\|^{2}=\|f\|^{2}-\left\|f-\chi_{(-\tau, \tau)} f\right\|^{2} \geqslant 1-\frac{M^{2}}{2 \tau} e^{-2 \tau^{2}} .
$$

Consequently, for all sufficiently large $\tau$ 's,

$$
\left\|\chi_{(-\tau, \tau)} f\right\| \geqslant\left(1-\frac{M^{2}}{2 \tau} e^{-2 \tau^{2}}\right)^{\frac{1}{2}} \geqslant 1-\frac{M^{2}}{2 \tau} e^{-2 \tau^{2}}
$$

and

$$
\arccos \left\|\chi_{(-\tau, \tau)} f\right\| \leqslant \arccos \left(1-\frac{M^{2}}{2 \tau} e^{-2 \tau^{2}}\right) .
$$

We recall that as $x \rightarrow 0^{+}$,

$$
\arccos (1-x)=\sqrt{2 x}(1+\mathcal{O}(x))
$$

Combining (72) and (173), we conclude that for every sufficiently large $\tau$

$$
\arccos \left\|\chi_{(-\tau, \tau)} f\right\| \leqslant \frac{2 M}{\sqrt{\tau}} e^{-\tau^{2}} .
$$

Similarly, for every sufficiently large $\omega$

$$
\arccos \left\|S_{\omega} f\right\| \leqslant \frac{2 M}{\sqrt{\omega}} e^{-\omega^{2}}
$$

Combining (74) and (75), and setting $\tau=\omega$, we obtain

$$
\arccos \left\|\chi_{(-\omega, \omega)} f\right\|+\arccos \left\|S_{\omega} f\right\| \leqslant \frac{4 M}{\sqrt{\omega}} e^{-\omega^{2}} .
$$


Setting $\Omega=\omega$ and $T=2 \omega$ in (69), we obtain

$$
\arccos \sqrt{\lambda_{0}\left(\omega^{2}\right)} \leqslant \arccos \left\|\chi_{(-\omega, \omega)} f\right\|+\arccos \left\|S_{\omega} f\right\| .
$$

Consequently,

$$
\arccos \sqrt{\lambda_{0}\left(\omega^{2}\right)} \leqslant \frac{4 M}{\sqrt{\omega}} e^{-\omega^{2}} .
$$

Substituting (64) into (65), we obtain

$$
\sqrt{\lambda_{0}\left(\omega^{2}\right)}=1-2 \sqrt{\pi} \omega e^{-2 \omega^{2}}\left(1+\mathcal{O}\left(\frac{1}{\omega^{2}}\right)\right) .
$$

Substituting (79) into (73), we obtain

$$
\arccos \sqrt{\lambda_{0}\left(\omega^{2}\right)}=2 \sqrt[4]{\pi} \sqrt{\omega} e^{-\omega^{2}}\left(1+\mathcal{O}\left(\frac{1}{\omega^{2}}\right)\right) .
$$

Combining (78) and (80), we arrive at a contradiction

$$
2 \sqrt[4]{\pi} \sqrt{\omega} e^{-\omega^{2}}\left(1+\mathcal{O}\left(\frac{1}{\omega^{2}}\right)\right) \leqslant \frac{4 M}{\sqrt{\omega}} e^{-\omega^{2}} .
$$

Our proof of Theorem 1.2 uses some techniques similar to those in [5], e.g. a linear combination of the time and the frequency limiting operators is already considered in [5, equation (6)].

\section{Acknowledgements}

The authors thank the reviewers for their helpful comments and suggestions, which have greatly improved this paper. The authors are supported by the FWF grants S10602-N13 and P23902-N13.

\section{References}

[1] F. Bonsall and J. Duncan. Complete Normed Algebras. Springer-Verlag, New York, 1973.

[2] M. Cowling, L. Escauriaza, C. E. Kenig, G. Ponce, and L. Vega. The Hardy uncertainty principle revisited. Indiana Univ. Math. J., 59:20072026, 2010. 
[3] L. Escauriaza, C. E. Kenig, G. Ponce, and L. Vega. The sharp Hardy uncertainty principle for Schrödinger evolutions. Duke Math. J., 155(1):163187,2010 .

[4] G. H. Hardy. A theorem concerning Fourier transforms. J. London Math. Soc., 8(3):227-231, 1933.

[5] Landau H. J. and H. O. Pollak. Prolate spheroidal wave functions, Fourier analysis and uncertainty - II. Bell System Technical Journal, 40:65-84, 1961.

[6] Petter E. Bjørstad and Jan Mandel. On the spectra of sums of orthogonal projections with applications to parallel computing. BIT, 31:76-88, 1991.

[7] D. Slepian. Some comments on Fourier analysis, uncertainty and modeling. SIAM Rev., 25:379-393, 1983.

[8] D. Slepian and E. Sonnenblick. Eigenvalues associated with prolate spheroidal wave functions of zero order. Bell Systems Tech. J., 44:17451758, 1965.

[9] T. Tao. Hardy's uncertainty principle. Website, 2009. http://terrytao.wordpress.com/2009/02/18/hardys-uncertainty-principle. 